\title{
Médiation(s) du lieu littéraire et figure(s) de Marcel Proust
}

Mediation(s) of the Literary Place and Figure(s) of Marcel Proust

Delphine Saurier

\section{OpenEdition}

Journals

Édition électronique

URL : https://journals.openedition.org/recherchestravaux/2257

DOI : 10.4000/recherchestravaux.2257

ISSN : 1969-6434

Éditeur

UGA Éditions/Université Grenoble Alpes

Édition imprimée

ISBN : 978-2-37747-197-3

ISSN : 0151-1874

Référence électronique

Delphine Saurier, « Médiation(s) du lieu littéraire et figure(s) de Marcel Proust », Recherches \& Travaux [En ligne], 96 | 2020, mis en ligne le 23 juin 2020, consulté le 29 juin 2021. URL : http://

journals.openedition.org/recherchestravaux/2257 ; DOI : https://doi.org/10.4000/recherchestravaux. 2257

Ce document a été généré automatiquement le 29 juin 2021.

(c) Recherches \& Travaux 


\title{
Médiation(s) du lieu littéraire et figure(s) de Marcel Proust
}

\author{
Mediation(s) of the Literary Place and Figure(s) of Marcel Proust
}

Delphine Saurier

1 Mais qu'est-ce donc que «le patrimoine littéraire »? On y trouve les œuvres, les auteurs, les livres ou encore les lieux historiques fréquentés par les écrivains. On se questionne alors sur la remédiatisation du patrimoine littéraire par le livre enrichi ${ }^{1}$, sur les enjeux de formation qu'il suppose ${ }^{2}$, sur le rôle des bibliothèques dans la constitution du patrimoine littéraire contemporain ${ }^{3}$, sur l'écrivain entrant au Panthéon et par la même occasion dans le cercle du patrimoine national, sur les maisons d'écrivain comme nouveau patrimoine ${ }^{4} . .$. Le patrimoine littéraire n'est plus un corpus consolidé d'œuvres littéraires passées à la postérité. Il est une œuvre, un auteur, un bâtiment. Il mobilise une foule d'acteurs qui travaillent de plus en plus à répondre à la « flottabilité de l'intérêt des publics ${ }^{5}$ ", voire il est pensé par le public ${ }^{6}$. Il suppose une multiplication d'ancrages qui participent à sa patrimonialisation. Comment, dès lors, saisir le patrimoine littéraire?

2 Dans le courant des années 2000, les études et recherches entreprises sur les lieux historiques consacrés aux personnes célèbres sont essentiellement axées sur le cas particulier des maisons d'écrivain. La bibliographie qui en résulte témoigne de deux logiques : des monographies ${ }^{7}$ très précises côtoient des tentatives de dresser une histoire française ${ }^{8}$ voire européenne de cette catégorie de lieux. Cette dialectique est à l'œuvre dans les travaux qui sont consacrés plus globalement au patrimoine et aux processus de patrimonialisation ${ }^{9}$. En effet, d'un côté, certaines recherches ${ }^{10}$ mettent en évidence l'importance des actes officiels (décrets, circulaires, etc.) qui sanctionnent l'objet culturel à un moment donné de son histoire, lui accordant par là même un nouveau statut, une reconnaissance, une garantie de préservation. Ce sont les grandes articulations qui sont pointées, offrant une histoire générale du patrimoine. On est alors tenté de comprendre la patrimonialisation comme un mouvement logique et raisonné, résultant de la démarche d'acteurs bien identifiés et qui opèrent selon une grille de critères modélisée. Mais d'un autre côté, certaines études ont montré que, 
derrière ces actes officiels, se trouvent les professionnels ou les amateurs en charge de l'objet culturel, susceptibles de faire émerger et évoluer le patrimoine selon des motivations qui leur sont personnelles ${ }^{11}$. Ce sont généralement des terrains particuliers qui sont investis, démarche d'où émergent des monographies dans lesquelles sont finement décortiquées les interactions entre acteurs directs. Dans ce cas, la patrimonialisation apparaît plutôt comme la résultante d'un parcours aléatoire, spécifique à l'objet, en tant qu'il est ancré dans un contexte qui lui est propre ${ }^{12}$.

Cetribuer à dépasser ces deux logiques. Pour cela, il reprend et discute une recherche portant sur la patrimonialisation de lieux historiques consacrés à des personnes célèbres ${ }^{13}$ et développée dans une perspective communicationnelle. Nous présenterons dans un premier temps les résultats de la recherche, pour envisager ensuite la façon dont l'exploration du terrain a mobilisé des acceptions différentes du concept de médiation. Enfin, nous proposerons une articulation entre cette notion de médiation et celle de figure. Ce faisant, cet article est avant tout centré sur une réflexion méthodologique et conceptuelle qui s'impose dès lors que l'on porte son regard sur les formes ancrées de la littérature, notamment les lieux historiques consacrés à des personnes célèbres.

\section{Patrimonialisation de lieux historiques : perspective comparée}

4 Deux terrains ont initialement été analysés, deux lieux historiques consacrés aux personnes célèbres : la Maison de tante Léonie-Musée Marcel Proust, située à IlliersCombray (Eure-et-Loir), maison des vacances enfantines de Marcel Proust, et le musée Curie (Paris, $5^{\mathrm{e}}$ arrondissement), qui offre à la visite un espace d'exposition, le bureau et le laboratoire personnel de Marie Curie. L'analyse réalisée dans une perspective historique et comparatiste devait permettre de mettre en évidence les étapes de la patrimonialisation tout en identifiant les caractéristiques propres au patrimoine littéraire. Elle a montré trois temps de vie des lieux.

5 Le premier temps est celui de la création des associations, initialement structurées autour des personnes admirées : la Société des Amis de Marcel Proust et des Amis de Combray (SAMPAC) et l'Association Frédéric et Irène Joliot-Curie (AFIJC, devenue ensuite l'Association Curie et Joliot-Curie) sont deux associations mémorielles, des rassemblements d'admirateurs, d'amis et de membres de la famille autour d'une personnalité littéraire et de personnalités scientifiques. À sa création en 1950, la SAMPAC construit et diffuse l'image d'une œuvre littéraire - À la recherche du temps perdu - susceptible de révéler l'individualité de son lecteur tout en développant sa compréhension du monde, mais également d'enrichir le « trésor intellectuel humain ${ }^{14}$ » de l'humanité. Ces pouvoirs attribués à l'œuvre légitiment la création de l'association, structurent sa vocation philanthropique et justifient sa volonté de territorialiser la mémoire de Marcel Proust et de son œuvre : un ancrage qui passe par la préservation d'une maison dans laquelle Marcel Proust a séjourné étant enfant. Quant à l'AFIJC, créée en 1959 sous l'impulsion de la famille et des amis de Frédéric Joliot-Curie, elle élabore d'emblée des figures du couple Joliot-Curie exceptionnelles et exemplaires. Ces caractéristiques sont fondées sur les découvertes scientifiques certes, mais surtout sur les engagements sociaux et politiques des deux chercheurs. Elles viennent justifier le fait qu'une association rende hommage au couple de scientifiques : c'est dans son cadre 
que les membres partagent des souvenirs personnels ; c'est à partir de son cadre qu'ils souhaitent développer des dispositifs de commémoration en direction de tous. Le bureau et le laboratoire des scientifiques, préservés, constituent l'un de ces dispositifs. La volonté mémorielle qui motive ces associations fait alors apparaitre une unité trompeuse : si leur désir de préserver la mémoire de personnalités les rassemble, leur travail sur la mémoire diffère largement, sur deux points au moins. D'une part, l'œuvre littéraire est l'instrument de légitimation des démarches de la SAMPAC, quand la personnalité des chercheurs est celui de l'AFIJC. D'autre part, l'objectif de la SAMPAC est moins la diffusion de l'œuvre que l'épanouissement des individus; en revanche l'utilité de l'AFIJC est locale, son objet est la science, sa diffusion et le renouvellement de sa communauté. Ces divergences de missions et d'idéologies se traduisent directement dans les démarches de préservation des lieux historiques, leurs mises en forme et leurs modalités d'ouverture aux visiteurs.

Dans un deuxième temps, les lieux historiques préservés deviennent des espaces de conservation de mémoires ${ }^{15}$, assurant matérialité et visibilité aux personnes admirées érigées en personnalités admirables. D'un côté se trouve un lieu de vie et de villégiature pour Marcel Proust, la maison de l'oncle Amiot rebaptisée "Maison de tante Léonie ", du nom de la tante du narrateur d'À la recherche du temps perdu; de l'autre côté se dresse un lieu de travail et de recherche, le bureau et le laboratoire personnel de Marie Curie, qu'occuperont sa fille, Irène et son gendre, Frédéric Joliot-Curie. Ces lieux littéraires et scientifiques sont perçus comme une forme de matérialisation de la mémoire concernant les personnes honorées: ce sont des traces qui attirent le respect. Or, l'arrivée de nouveaux occupants fait craindre la dégradation de ces traces et engage à un élan pour la sauvegarde des deux lieux. L'intérêt pour la maison de Jules Amiot est tardif, en ce sens qu'elle a connu les réaménagements successifs d'occupants après la mort de l'oncle. La SAMPAC fixe alors d'emblée la nécessité d'une reconstitution du décor qu'a pu connaître Marcel Proust, mais aussi le petit Marcel de l'œuvre fictionnelle, dans l'objectif de faire accéder tous les individus à l'art et à une compréhension d'euxmêmes, selon le principe philanthropique au fondement de l'association. Toutefois, de façon concomitante, selon les membres de la SAMPAC, le caractère sacré et cultuel des lieux craint toute appropriation massive. Sont alors préférées les démarches de recueillement et les attitudes pèlerines : les membres de l'association s'attachent au respect des lieux qui requièrent, selon eux, une pratique de " dévot ». Ainsi, l'ouverture de la Maison de tante Léonie et la figure de Marcel Proust sont portées à la fois par une volonté de type messianique - qui induit une révélation de Marcel Proust auprès « $d u$ plus grand nombre d'individus» - et par une perspective corporatiste et élitiste réduisant l'accès à la compréhension de Marcel Proust et de son œuvre à un corps d'individus composé de «fervents admirateurs ». Ainsi, d'un espace privé, on fait un lieu public ouvert officiellement à tous, mais auquel on adjoint un caractère cultuel, qui rétrécit en retour ses possibilités d'accès. Le laboratoire personnel et le bureau de Marie Curie connaissent un mouvement inverse : d'un lieu de travail de la communauté scientifique construit sur un site universitaire appartenant à l'État, on fait un lieu privé, au sens où la famille contrôle selon ses souhaits son accès et préserve une mémoire. Quant à l'espace d'exposition qui lui est adjoint dans les premières années, il est réalisé par et pour des scientifiques, et ouvert sur demande: son accès reste confidentiel et très largement restreint. Il est possible de voir là une forme de contradiction avec l'une des missions que s'impose l'AFIJC, qui est de faciliter l'accès aux sciences. En somme, qu'il s'agisse de la Maison de tante Léonie ou du musée Curie, 
les actes de préservation dont ils font l'objet extraient les lieux de la vie quotidienne, des histoires qui s'écoulent, pour les placer dans des espaces mémoriels privés, qui cohabitent avec plus ou moins de bonheur et de restrictions avec la sphère publique. Au centre de ces nouveaux dispositifs se trouvent des personnalités admirables, dont les membres des associations dessinent les contours, s'appuyant pour cela sur un terrain ancien : l'exaltation de l'homme hors du commun.

Enfin, un troisième temps est celui de la consécration des personnalités admirables en Illustres. Les portraits de l'écrivain et des scientifiques vont progressivement s'affiner dans les structures associatives et prendre forme dans la matérialité des lieux de mémoire. Ils renvoient à des modèles idéaux anciens caractéristiques du poète-écrivain pour Marcel Proust - le génie, le sacré - et de l'homme de science pour Frédéric et Irène Joliot-Curie - le savant, le grand homme. Par-delà ces particularités qui les spécifient, ces personnes sont dignes d'être honorées pour ce qu'elles sont pour les hommes et donc pour ce qu'elles apportent à l'humanité : Marcel Proust a, en tant que guide, une valeur prophétique ; Frédéric et Irène Joliot-Curie ont, en tant que modèles, une valeur d'exemplarité. Tout élément biographique propre à l'écrivain et aux scientifiques est susceptible de légitimer l'utilisation de ces modèles idéaux qui, une fois convoqués, affermissent, voire enrichissent le socle symbolique des personnes admirées dans les cadres associatifs. Ce socle n'est donc pas constitué que des seuls modèles idéaux anciens : l'écrivain ${ }^{16}$ et les deux scientifiques, avec leur entourage biographes et autres commentateurs ${ }^{17}$ - participent à la construction de portraits. L'ensemble de ce matériau permet aux membres des associations de renforcer progressivement certaines caractéristiques (l'écriture dans la maladie, la dimension cathartique de l'œuvre, l'idéalisme scientifique, l'humanisme), d'effacer des traces (les conséquences négatives de la radioactivité, l'homosexualité de Marcel Proust, l'adhésion au communisme des Joliot-Curie) pour leur substituer de nouvelles facettes (l'intellectuel engagé, la responsabilité politique du scientifique, la sainte et la féministe). Ce travail associatif sur les portraits des illustres fait écho aux évolutions culturelles, sociales et politiques : celles des courants d'études littéraires, de l'histoire des sciences, de la communauté des historiens ${ }^{18}$, des politiques publiques, des mouvements politiques... Avec le temps, on déleste donc les portraits de certaines valeurs pour leur en conférer d'autres qui leur permettent de circuler dans différents espaces sociaux avec efficacité et fluidité : Marie Curie, femme de sciences précurseur et dévouée, circule indifféremment et avec aisance dans les associations féministes, au CNRS, à la fondation Curie, dans la sphère politique ; Marcel Proust, architecte de génie d'une œuvre-cathédrale, circule avec force dans les manuels scolaires, les laboratoires de recherche universitaire, les devantures de librairies, la Bibliothèque nationale de France et encore les hors-séries estivaux des magazines. Ce faisant, la personne admirable devient un illustre ${ }^{19}$ et un être culturel ${ }^{20}$ : naturalisée dans les espaces communs, elle se retrouve sans faire débat dans les manuels d'histoire, de sciences et de littérature, dans les discours politiques, dans les œuvres fictionnelles... dans les maisons d'Illustre et les « chemins » touristiques. Un renversement des rapports entre lieux et figures d'illustres s'opère : les figures signifient plus les lieux qu'elles ne sont signifiées par eux. Autrement dit, la construction des figures d'illustres échappe à l'emprise des lieux et de leur domaine premier d'appartenance. Ces figures deviennent des êtres culturels par excellence de la trivialité, échappant à la seule sphère patrimoniale ${ }^{21}$. Ce sont des «mixtes d'objets, de représentations et de pratiques [qui] fournissent aux formes culturelles d'une société leurs repères d'interprétation et de 
valeur ; ils servent d'opérateurs pour les représentations du monde, mais aussi pour les postures culturelles et les catégories de jugement qu'une culture peut reconnaitre ${ }^{22}$. »

\section{Patrimoine littéraire et médiation(s)}

Comment arrive-t-on à une telle analyse des ancrages du patrimoine littéraire - par le prisme de lieux historiques - et que nous dit-elle de la patrimonialisation de la matière littéraire? Nous proposons d'envisager cette question en ouvrant la boîte noire de notre « cuisine ${ }^{23}$ » de chercheur, entremêlant démarche méthodologique et réflexion conceptuelle.

Partant initialement d'une volonté de confronter le discours patrimonial d'un site à sa réception, nous avons d'abord porté notre attention sur une médiation culturelle - le discours du guide conférencier - entendue comme un acte ou un outil produit par l'institution culturelle et ses acteurs ${ }^{24}$. La visite proposée, au contenu accessible à un public d'avertis, est animée de façon vivante et personnelle. Le temps est aboli pour faire revivre au visiteur l'époque de Marcel Proust, le passé est actualisé et l'émotion mobilisée. Le propos développé mêle intimement des informations sur l'auteur et des récitations de longs passages d'À la recherche du temps perdu, mettant en scène des personnages de l'œuvre, essentiellement «le petit Marcel ». L'intention explicitée ${ }^{25}$ de ce type de visite est de permettre au visiteur d'appréhender la démarche créatrice par confrontation du texte au lieu historique. Cette intention implique pour le chercheur de dépasser le cadre interprétatif que constitue la bipartition entre le guide conférencier-producteur et le visiteur-récepteur: la Maison de tante Léonie, en tant qu'espace muséographié, doit elle-même être interrogée comme une médiation. Pour ce faire, nous nous sommes adossés à la compréhension du dispositif médiatique tel qu'envisagé par Jean Davallon à propos de l'exposition. Selon cet auteur, le dispositif médiatique peut être considéré comme un média au sens d'« objet culturel », c'est-àdire, « un objet construit que l'on visite, résultant d'une production formelle et destiné à produire chez celui qui les reçoit un "effet" symbolique ${ }^{26}$ ». L'analyse de la muséographie de la Maison de tante Léonie montre que celle-ci double - ou est doublée par - l'ambiguïté du propos développé par le guide conférencier: sans cordon, ni barrière, ni panneau scriptovisuel, la muséographie du lieu propose une expérience immersive dans un espace signifiant tout autant l'écrivain que le petit Marcel d'À la recherche du temps perdu. Dans ce cadre, le visiteur est à la fois « objet des stratégies de l'instance de production et sujet de stratégies d'appropriation ${ }^{27} »$ : ce constat nous incite alors à réaliser des entretiens avec les visiteurs afin de confronter le discours produit à sa réception. Dans un premier temps, il nous semble que la réception réponde parfaitement à l'intention du message, puisque les entretiens montrent que l'ambiguïté des lieux conduit les visiteurs à s'intéresser au processus de création. Le dispositif que constitue la Maison de tante Léonie remplirait alors son objectif. Néanmoins, cet intérêt des visiteurs pour le processus de création, compris dans la confrontation entre l'auteur et le petit Marcel, se retrouve dans les propos des visiteurs interrogés de l'exposition «Marcel Proust ${ }^{28}$ » à la Bibliothèque nationale de France, alors même qu'ils ne connaissent pas la Maison de tante Léonie. Cet intérêt n'est donc pas nécessairement le fruit de la rencontre avec le dispositif du lieu historique. Elle peut lui être préalable. Allons plus loin, elle est commune aux admirateurs des Illustres si l'on se réfère aux entretiens réalisés avec les visiteurs du musée Curie qui cherchent à s'approcher au 
plus près du génie découvreur ${ }^{29}$. L'exploration du terrain fait éclater notre cadre conceptuel de la médiation comme dispositif considéré sur un plan synchronique et nous permet d'opérer un double déplacement: l'objet de la recherche n'est plus la bipartition production/réception analysée dans le cadre de la médiation culturelle, mais les processus communicationnels par lesquels passe la patrimonialisation du lieu historique littéraire ; les formes qui retiennent l'attention ne sont plus la médiation culturelle comme dispositif médiatique, mais la multitude de ce par quoi passent les ancrages du littéraire. Ceux-ci s'apparentent aux médiations telles que définies par Antoine Hennion :

Si l'on entre dans les controverses réelles qui accompagnent la construction d'un goût, on n'a jamais affaire au sujet avec un grand $\mathrm{S}$ ni à l'objet avec un grand $\mathrm{O}$, mais à une procession hétéroclite de médiations, plus ou moins profondément inscrites dans de la matière et plus ou moins reconnues et partagées, toutes situées dans l'entre-deux qui va des humains aux choses ${ }^{30}$.

Et encore :

En modifiant leur définition, c'est ce que nous retenons des «mondes de l'art » de Becker : il n'existe aucun espace global qui serait celui de la musique. Comme les musiciens, nous n'avons accès à la musique qu'en entrant dans l'une de ces poches, un de ces «mollusques de référence » à la Einstein que sont les musiques réelles. En rassemblant des choses et des humains et en travaillant sur leur prise croisée, elles réussissent à se tracer une enveloppe suffisamment résistante pour retenir les éléments, et suffisamment souples pour s'agrandir, se modifier, se coller à des ensembles plus vastes - et dresser les axes de la représentation ${ }^{31}$.

10 Dès lors, nous cherchons à appréhender les "éléments " qui constituent l'«enveloppe», la "poche», le "mollusque de référence» qu'est la Maison de tante Léonie. Il n'est plus exclusivement question de dispositif tel que défini par les professionnels en tant que technique, mais aussi de processus de médiation qui soutiennent, définissent et modifient continuellement la Maison de tante Léonie ${ }^{32}$. Se pencher ainsi sur les processus de médiation invite à adopter une perspective sur le temps long et à élargir le spectre des éléments étudiés. Un ensemble composite de données est alors progressivement constitué regroupant des observations des visites guidées, l'analyse des discours du guide, l'étude de la muséographie des lieux, des entretiens avec les responsables associatifs et avec les visiteurs, l'analyse des archives de l'association, l'étude des articles de Marcel Proust concernant l'écrivain, etc. Comment approcher ce composite de données hétérogènes dans l'objectif de suivre l'évolution de la Maison de tante Léonie? Le travail de production des données a permis d'identifier deux éléments particulièrement structurants pour les processus de patrimonialisation des lieux : le «public » et «Marcel Proust ». L'analyse s'est concentrée sur ces deux médiations et s'est développée par croisement de trois échelles de contexte $^{33}$ et de trois dimensions ${ }^{34}$.

\section{Ancrages du littéraire, figures et opérativité symbolique des médiations}

11 Ainsi comprise, la médiation revêt trois caractéristiques essentielles dans cette démarche : elle permet l'identification de tout ce qui intervient dans le processus de patrimonialisation, sans classification préalable; elle permet d'envisager la rencontre de ce qui intervient dans la patrimonialisation sans définir a priori les relations qui les lient, sans nier a posteriori les positions éventuellement déséquilibrées qui les 
contraignent; elle permet d'envisager le tout qui dépasse ses parties et qui fait cohérence sociale et politique avec ou sans ses parties. S'intéresser à la patrimonialisation de lieux historiques à l'aide du concept de médiation revient alors à se pencher sur "ce qui fait évoluer", c'est-à-dire les éléments qui composent le patrimoine et leurs liens. Selon cette perspective, le lieu littéraire est le fruit d'interventions multiformes perméables aux contextes historiques et sociaux: il est alors une forme instable, néanmoins consolidée autour d'un socle représentationnel. Mobiliser le concept de médiation permet, ce faisant, de sortir d'une compréhension clivée de la patrimonialisation : fruit des structures et des institutions ou des actions des individus. Malgré cet apport essentiel de la médiation, par la dimension processuelle mise à l'honneur, l'opérativité symbolique du patrimoine a tendance à passer au second plan. C'est en raison, nous semble-t-il, du caractère englobant de la notion: la médiation peut revêtir tous types de formes (individu, objet, représentation...), elle est évolutive et instable tout en étant considérée dans son unicité. C'est précisément ce qui fait de la médiation un quasi-concept si l'on suit Christine Servais qui emprunte l'expression à Jacques Derrida. Selon elle, le quasiconcept marque «le fait que ces concepts [comme la médiation] se défont eux-mêmes car ils emportent avec eux la dimension empirique des phénomènes, et donc la variabilité infinie de ceux-ci, mais également leur possible impossibilité ${ }^{35} »$. Afin de descendre en finesse dans la compréhension de l'opérativité symbolique du patrimoine, il s'est alors agi pour nous d'appareiller ce quasi-concept de médiation à la notion de figure.

De quelle façon la figure de Marcel Proust fait-elle médiation dans le cadre de la patrimonialisation de la Maison de tante Léonie? De fait, Marcel Proust est en soi une figure, mais il est également la figuration de toute une série de figures: c'est une " figure d'illustre ", une " figure d'auctorialité », une « figure exemplaire », une " figure de la mondanité ", une figure métaphorique renvoyant à l'œuvre, etc.: depuis quinze ans, nos travaux reprennent ainsi toutes ces figures et illustrent la variabilité de l'usage de la notion en sciences humaines et sociales ${ }^{36}$. Cette variabilité, qui prend racine dans le caractère double de la figure, en fait toute la richesse heuristique. Cette duplicité se retrouve parfaitement dans la figure de Marcel Proust : il incarne le grand écrivain compris comme une figure visible à la surface du tableau social qui, dans le même temps, dissimule les valeurs, les représentations, les savoirs construits au prix de désaccords et consensus. Aussi, la figure de Marcel Proust est un "opérateur " permettant au chercheur de se placer à l'endroit du « clivage entre ce qui est en train d'advenir mais qui n'est pas inscrit, et ce qui est inscrit et a trouvé forme ${ }^{37}$ ».

Ce que le tableau représente n'est pas ce qu'il figure [...]. Les figures du tableau sont, en quelque sorte, à sa surface comme les index de cette représentation: elles désignent le monde, pour nous spectateurs, mais en même temps renvoient à l'espace épistémologique configuré dont le tableau est le représentant. Sur ce plan, les figures explicites du tableau s'organisent en niveau symbolique. En semblant, dans ses figures, désigner le monde - c'est là le leurre de la figure comme image des choses - le tableau n'est pas autre chose que l'espace de représentation où les systèmes qui le constituent s'implicitent. Le leurre de la figure comme image dissimule le savoir qui se représente dans le tableau ${ }^{38}$.

13 Chercher à appréhender la duplicité de la figure ${ }^{39}$ revient à dépasser le leurre iconique qui conduit à désigner les auteurs comme des incarnations de l'auctorialité, du créateur ou encore du génie, pour chercher à rendre lisibles les « systèmes ", le « savoir » qui s'y implicitent. Une perspective que peine à développer le quasi-concept de médiation. Se 
focaliser sur les figures permet d'envisager les questionnements suivants : comment et pourquoi Marcel Proust contribue-t-il à ancrer l'auctorialité et le génie dans des lieux historiques et plus globalement dans le monde littéraire? Et comment ces figures d'écrivain nous parlent-elles plus globalement de notre société ?

\section{Conclusion}

Nous avons souhaité approcher la patrimonialisation de la littérature par le biais de l'analyse des rapports qui se jouent entre producteurs et récepteurs de la médiation culturelle, via l'étude de dispositifs de médiation humaine et de l'expographie. Si, dans un contexte muséographique de montée en puissance des idées de co-construction et de participation, notre regard était critique, notre posture sur le terrain était, elle, plutôt de nature pragmatique. Aussi, avons-nous progressivement élargi l'investigation au-delà des dispositifs de médiation compris sur un plan synchronique initialement ciblés. Ce faisant, la médiation n'est plus attachée exclusivement aux techniques professionnelles ou au "tiers symbolisant ${ }^{40}$ ", elle ne suppose pas d'abord de projet démocratique spécifique ${ }^{41}$, elle est un concept permettant l'identification, l'observation et l'analyse de ce qui fait advenir - mais aussi de ce qui freine - l'être culturel auquel on s'intéresse ${ }^{42}$. Considérer ainsi la médiation, c'est libérer le chercheur comme le professionnel de l'injonction performative et de la mesure de l'atteinte du projet démocratique ${ }^{43}$, c'est se donner la possibilité de prendre en filature, selon les chemins qu'ils empruntent, les projets, intentions, prétentions au fondement des actions et des mises en lien qui façonnent l'être culturel. C'est ainsi que nous avons pu étudier la patrimonialisation de la Maison de tante Léonie, prise dans des courants culturels de fond (l'admiration pour l'homme illustre par exemple), des paradigmes scientifiques (histoire littéraire vs génétique notamment), des politiques publiques (comme la labellisation des « Maisons des Illustres » et l'éducation artistique et culturelle...), des tensions organisationnelles et portée par des acteurs dont les socialisations ne sont pas sans effet. Le concept de médiation nous a donc engagés à identifier le réseau de ce qui fait émerger le patrimoine (comment cela fonctionne-t-il ? ([qu'est-ce] qui est engagé dans ce réseau ?) Si la médiation permet également de voir qu'il se joue des choses, on peine néanmoins à analyser finement son opérativité symbolique. Tandis qu'observer la figure de Marcel Proust, qui fait médiation au cours de la patrimonialisation de la Maison de tante Léonie, revient à s'attarder, de façon focalisée mais non statique, sur un élément doué de duplicité : prendre au sérieux cette caractéristique implique de regarder comment la figure, en étant visible, in-visibilise la matière sociale et le fonds culturel qui la soutiennent. 


\section{NOTES}

1. A. Laborderie, C. Jeantet, $P$. Hellégouarc'h, « Remédiatiser le patrimoine littéraire à travers le livre enrichi: quelles expériences de lecture?», Revue de recherches en littératie médiatique multimodale, 8 août 2018.

2. B. Louichon, «Le patrimoine littéraire : un enjeu de formation », Tréma, $\mathrm{n}^{\circ} 43,2015$, p. 22-31.

3. Question explorée lors d'une table ronde « Le rôle des bibliothèques dans la constitution du patrimoine littéraire contemporain ", d'une journée professionnelle franco-luxembourgeoise, Les enjeux transfrontaliers de la filière du livre, abbaye de Neumünster, Luxembourg, 7 mars 2019.

4. D. Fabre, « Maison d'écrivain. L'auteur et ses lieux », Le Débat, vol. 115, n 3, 2001, p. 172-177.

5. Le propos est développé par Éric Dussert, invité à débattre lors d'une Journée professionnelle franco-luxembourgeoise, Les enjeux transfrontaliers de la filière du livre, abbaye de Neumünster, Luxembourg, 7 mars 2019.

6. C'est le cas de nombreux projets participatifs autour du patrimoine, comme celui de « Nantes Patrimonia » (<https://patrimonia.nantes.fr/home/a-propos-1.html>).

7. Certains sites ont fait l'objet d'articles ou de petites monographies que l'on doit généralement aux individus responsables de la conservation des lieux. Consulter pour exemple Judith MeyerPetit («La maison de Balzac et les paradoxes du musée littéraire », Revue d'histoire littéraire de la France, $\mathrm{n}^{\circ} 1$, janvier-février 1995). Voir également J. Gribenski, V. Meyer, S. Vernois (dir.), La Maison de l'artiste, Rennes, PUR, 2007.

8. G. Poisson, Les Maisons d'écrivains, PUF, coll. « Que sais-je ? », Paris, 1997.

9. À noter toutefois l'émergence de deux démarches plus novatrices : la réflexion développée par Jean Davallon (Le don du patrimoine : une approche communicationnelle de la patrimonialisation, Paris, Hermès Science-Lavoisier, 2006), en sciences de l'information et de la communication, qui cherche à dégager une logique de patrimonialisation commune aux objets patrimoniaux, qui serait celle du don ; la démarche de Nathalie Heinich (La Fabrique du patrimoine. De la cathédrale à la petite cuillère, Paris, Maison des Sciences de l'Homme, séries « Ethnologie de la France ", 2009) qui reste profondément ancrée au terrain et aux objets pour saisir les opérations et procédures génériques qui façonnent les valeurs du patrimoine.

10. Des recherches essentiellement historiques (avec D. Poulot [dir.], Patrimoine et modernité, Paris, L'Harmattan, 1998), notamment, ont proposé une histoire générale du patrimoine français. D'autres comme Krzysztof Pomian (Collectionneurs, amateurs et curieux: Paris, Venise, XVI', $\mathrm{XVIII}^{e}$ siècle, Paris, Gallimard, 1987) se sont axés sur l'analyse fine d'une période plus courte, quand certains se sont intéressés à des aspects plus particuliers du patrimoine, comme celui des politiques patrimoniales (P. Poirrier, L'État et la culture en France au $\mathrm{xx}^{e}$ siècle, Paris, Librairie générale française, Le Livre de Poche, 2000).

11. L'analyse du site du Creusot par Octave Debary (La Fin du Creusot ou l'art d'accommoder les restes, Paris, Éditions du Comité des Travaux Historiques et Scientifiques, coll. «Le regard de l'ethnologue ", ministère de la Recherche, 2002) en est l'un des premiers exemples particulièrement aboutis.

12. J. Davallon (Le Don du patrimoine, ouvr. cité, p. 16) propose une autre répartition des études et recherches consacrées au patrimoine, qui auraient dans un premier temps considéré l'objet patrimoine selon sa nature et sa fonction. Suivant cette perspective, certains cherchent à identifier les qualités intrinsèques du patrimoine, servant en ce sens la volonté politique de modéliser et de normaliser cette catégorie; d'autres trouvent le sens du patrimoine dans les utilisations sociales qui en sont faites, faisant émerger une préoccupation nouvelle pour le public, que ce soit du point de vue de la recherche ou de celui des institutions patrimoniales. 
13. La première partie de cet article reprend des passages du livre: D. Saurier, La Fabrique des illustres : Proust, Curie, Joliot et lieux de mémoire, Paris, éditions Non Standard, coll. « SIC, », 2013.

14. Cette citation et les suivantes entre guillemets et en italique sont issues des bulletins de la SAMPAC.

15. Ce deuxième temps est donc celui de la stratification mémorielle des lieux, celui de la mémoire des lieux (P. Ricœur, La Mémoire, l'histoire, l'oubli, Paris, Le Seuil, coll. «Essais, », 2003, p. 736).

16. Se référer à $D$. Saurier, «La figure de l'auteur comme médiation littéraire. L'autoportrait de Marcel Proust ", dans A. Alves et M. Pourchet (dir.), Les Médiations de l'écrivain, Paris, L'Harmattan, 2011.

17. Consulter D. Saurier, "Marcel Proust selon des commentateurs "ordinaires" ", Communication et langages, PUF, $\mathrm{n}^{\circ} 150$, décembre 2006, p. 45-64.

18. On pense ici à la publication des Lieux de mémoire (Gallimard, 1984-1992) sous la direction de Pierre Nora et de la bonne fortune de l'expression dans les années 1980 et 1990 qui implique, dans ces années-là, une compréhension des lieux historiques consacrés aux personnes célèbres comme lieux de mémoire.

19. L'illustre peut ici être compris à la fois comme un lieu de mémoire au sens de Pierre Nora (Les lieux de mémoire, Paris, Gallimard [Bibliothèque illustrée des histoires], 3 tomes: t. 1: La République [1 vol., 1984], t. 2 : La Nation [3 vol., 1986], t. 3 : Les France [3 vol., 1992]) et comme un instrument institutionnel de gestion du patrimoine : il réfère à la fois à la galerie des Illustres et au label des « Maisons des Illustres » créé en 2011 par le ministère de la Culture.

20. Y. Jeanneret, dans Critique de la trivialité. Les médiations de la communication, enjeux de pouvoir (Paris, Éditions Non Standard, 2014, p. 12), définit l'être culturel comme un " ensemble d'idées et de valeurs qui incarne un objet de la culture dans une société tout en se transformant constamment à partir de la circulation des textes, des objets et des signes. L'image de la science, ou scientificité, joue un rôle déterminant dans notre société; elle se concentre sur des conceptions assez vagues mais insistantes de ce que sont la précision, la mesure, la rationalité, idées que les chiffres sont par exemple particulièrement aptes à incarner ».

21. Mais cette évolution n'est pas systématique et certaines figures échouent à l'examen : en effet, si la figure de Marcel Proust rejoint sans encombre le statut de grand écrivain, celle de Marie Curie est propulsée à la tête du musée Curie, contraignant la figure des Joliot-Curie à rester dans la sphère étroite de l'histoire de la physique nucléaire. On voit ici comment la médiation peut être "processus ou résultat selon que le mouvement de conflictualité s'y épuise ou s'y dynamise » (C. Servais, « Qu'est-ce que la médiation ? Examen critique d'une déconstruction de la communication", dans C. Servais [dir.], La Médiation. Théorie et terrains, Louvain la Neuve, De Boeck Supérieur, 2016, p. 159).

22. Y. Jeanneret, Penser la trivialité, vol. 1, La trivialité des êtres culturels, Paris, Hermès, 2008, p. 16.

23. Nous reprenons l'expression de Joëlle Le Marec dans son Habilitation à diriger les recherches (Ce que le "terrain» fait aux concepts : vers une théorie des composites, université Paris 7, 2002).

24. Pour une telle compréhension, se référer à É. Caillet et É. Lahalle (À l'approche du musée : la médiation culturelle, Lyon, PUL, 1995) ou à M.-C. Bordeaux (Médiations culturelles, formes communicationnelles et enjeux d'éducation non formelle, Mémoire d'Habilitation à diriger les recherches en sciences de l'information et de la communication, université d'Avignon et des pays du Vaucluse, 2015).

25. «Il y a quelques personnes discrètes et bénévoles qui offrent un peu de leur temps, de leur talent, des ressources de leurs greniers ou de leurs armoires pour décorer d'un bibelot, d'un napperon, d'un rideau, voire d'un meuble "proustien" un coin de la "Maison de Tante Léonie" qui doit vivre "dans le temps". ( (A. Borrel, "Vie de la Société », Bulletin de la Société des Amis de Marcel Proust et des Amis de Combray, 1993, $\mathrm{n}^{\circ}$ 43, p. 189). La Maison de tante Léonie, rassemblant des meubles authentiques et des décors inspirés par l'œuvre, est présentée comme « une sorte de 
synthèse des "lieux proustiens", ceux où Marcel Proust a vécu et ceux qu'il a rêvés et recréés à partir d'une réalité que le visiteur aura sous les yeux; et c'est bien là ce qu'on vient chercher dans une maison d'écrivain» (A. Borrel, "Vie de la Société », Bulletin de la Société des Amis de Marcel Proust et des Amis de Combray, 1994, $\mathrm{n}^{\circ}$ 44, p. 212).

26. J. Davallon, L'exposition à l'œuvre. Stratégies de communication et médiation symbolique, L'Harmattan, Paris, 1999, p. 43.

27. Ibid., p. 44.

28. Exposition «Marcel Proust, l'écriture et les arts » à la Bibliothèque nationale de France, du 8 novembre 1999 au 6 février 2000.

29. D. Saurier, J. Eidelman, Perception et réception, l'utilisation des formes de médiation au Musée Curie, deuxième partie: étude qualitative, CERLIS (CNRS/Paris 5), Musée Curie (CNRS/Institut Curie), septembre 2000.

30. A. Hennion, La passion musicale. Une sociologie de la médiation, Paris, Métailié, 1993, p. 73.

31. Ibid., p. 371.

32. Ce faisant, nous renouons avec la compréhension foucaldienne du dispositif.

33. Une première échelle pourrait être qualifiée de "macro » : les acteurs de la conservation de l'objet comme les publics puisent dans un substrat collectif, dans différents systèmes de penser, communs à eux tous, qui composent une mémoire collective. La thèse de la logique du don propre au patrimoine, développée par Jean Davallon (Le Don du patrimoine, Paris, HermèsLavoisier, 2006), se situe à cette échelle, tout comme la notion de régime d'historicité proposée par François Hartog (Régimes d'historicité. Présentisme et expériences du temps, Paris, Le Seuil, 2003). Il s'agit d'une première série de cadres imposés à la patrimonialisation. Une échelle "méso " renvoie aux cadres propres au collectif en charge de la sauvegarde de l'objet culturel. Ce collectif est soudé et organisé par une volonté et un intérêt communs et il perdure - et parfois s'institutionnalise dans et par rapport au contexte social multidimensionnel. Quel est ce collectif? Comment se définit-il ? Comment se structure-t-il ? Quels sont les cadres fondateurs qui légitiment le groupe et conséquemment, l'objet patrimonial? Là encore, les logiques développées orientent à la fois le travail sur la symbolique de l'objet culturel et la patrimonialisation. Une échelle «micro", enfin, prescrit d'autres logiques au processus étudié. Elle correspond aux contextes propres aux personnes en charge des objets, considérées en tant qu'individus. C'est l'homme pluriel (B. Lahire, L'Homme pluriel. Les ressorts de l'action, Paris, Nathan, 2001) que cette échelle d'analyse micro permet d'appréhender. L'idée n'est pas d'analyser les personnes en charge de l'objet culturel en tant que professionnels (ou experts) du patrimoine uniquement: ses autres expériences socialisatrices sont prises en compte, car ses rencontres avec d'autres univers sociaux sont toujours susceptibles de venir nourrir son regard et sa réflexion sur l'objet culturel.

34. Il s'agit des dimensions logistiques, sémiotiques, sociologiques identifiées par Yves Jeanneret pour comprendre la vie triviale des êtres culturels.

35. C. Servais, art. cité, p. 16.

36. Concernant l'objet qui nous intéresse, on trouve des figures de style, des figures médiatiques, des figures plastiques (L. Marin, «Le discours de la figure », Critique, $n^{\circ} 270,1969$, p. 953-971) et encore des figures d'auteur (C. Massol, A.-M. Monluçon, B. Ferrato-Combe [dir.], «Figures paradoxales de l'auteur ", Recherches et travaux, $\mathrm{n}^{\circ}$ 64, 2004 [en ligne: <https:// journals.openedition.org/recherchestravaux/1183?lang=es>]; M. Couturier, La Figure de l'auteur, Paris, Seuil, coll. « Poétique », 1995).

37. Joëlle Le Marec, Ce que le "terrain » fait aux concepts : vers une théorie des composites, université Paris 7, 2002, p. 59.

38. L. Marin, « Le discours de la figure », Critique, $\mathrm{n}^{\circ} 270,1969$, p. 958-959. 
39. Duplicité aux deux sens du terme : le fait d'être double; le fait d'avoir un comportement double variant selon les circonstances. Pour la figure, il s'agit d'être un élément intermédiaire capable de renvoyer à la fois à une matérialité et à une abstraction.

40. L. Quéré dans Des Miroirs équivoques. Aux origines de la communication moderne (Paris, Aubier Montaigne, 1982, p. 33), précise que l'« interaction sociale met nécessairement en jeu un "tiers symbolisant”, le pôle extérieur d'un neutre, qui, n'étant ni (pour) l'un, ni (pour) l'autre, et occupant une position de référence possible pour l'un et l'autre, les conjoint dans leurs différences ». Il ajoute que ce tiers symbolisant correspond au " pôle institutionnel ».

41. J. Caune, La Médiation culturelle. Expérience esthétique et construction du Vivre-ensemble, Grenoble, Presses universitaires de Grenoble, coll. « Communication médias et sociétés », 2017.

42. Nous renouons ici avec l'une des formes de la médiation, telles que définies par Jean-Luc Nancy, et qui se concentre sur «la déconstruction de la notion de place, à travers la pluralité originaire, où l'identité collective n'est pas soumise à un horizon de sens mais où "nous arrivons" » (Christine Servais, art. cité, p 144).

43. Ce qui ne signifie pas que le projet démocratique n'existe pas, mais qu'il est compris comme une finalité des acteurs, que l'opérativité symbolique de la médiation déborde généralement.

\section{RÉSUMÉS}

Mais qu'est-ce donc que «le patrimoine littéraire »? On y trouve les œuvres, les auteurs, les livres ou encore les lieux historiques fréquentés par les écrivains. Le patrimoine littéraire n'est donc plus un corpus consolidé d'œuvres littéraires passées à la postérité. Il est une œuvre, un auteur, un bâtiment. Il mobilise une foule d'acteurs et suppose une multiplication d'ancrages qui participent à sa patrimonialisation. Comment, dès lors, saisir le patrimoine littéraire ? Cet article souhaite envisager cette question en reprenant et discutant une recherche (La fabrique des Illustres, Saurier, 2013) portant sur la patrimonialisation de lieux historiques consacrés à des personnes célèbres et développée dans une perspective communicationnelle.

What is "literary heritage"? It includes works, authors, books and historical places frequented by writers. Literary heritage is no longer a consolidated corpus of literary works passed on to posterity. It is a work, an author, a house. It mobilizes a host of actors and requires a multiplication of anchorages that participate in its heritage. How, then, can literary heritage be grasped? This article seeks to address this issue by taking up and discussing research on the heritage of historical places dedicated to famous people and developed with a communicational perspective.

\section{INDEX}

Keywords : literary place, development of heritage, Marcel Proust, figure, mediation

Mots-clés : lieu littéraire, patrimonialisation, Marcel Proust, figure, médiation 


\section{AUTEUR}

\section{DELPHINE SAURIER}

Delphine Saurier est enseignant-chercheur à Audencia, Nantes. Ses recherches portent notamment sur la construction de figures fondées sur les représentations et les portraits d'hommes et de femmes célèbres - ou non. Concernant ce sujet, elle a publié un ouvrage intitulé La fabrique des Illustres aux éditions Non Standard en 2013. 